\title{
The FPAA Realization of Analog Predictive Circuit
}

\author{
György Györök \\ Óbuda University \\ Regional Education and Innovation Center \\ Budai Str. 45, H-8000 Székesfehérvár \\ Hungary \\ gyorok.gyorgy@bmf.roik.hu
}

\begin{abstract}
The programmable analog circuit (FieldProgrammable Analog Array), as a new component and a new technology appeared some twenty years ago. As a result of the continuous development, these devices programmed on a digital surface make an analog circuit topology with component parameters also given as programmable. FPAAs can be used for the realization of different functional units, circuits, circuit elements. The advantage of FPAAs in the field of reconfigurable circuit planning is significant. It is beneficial in self-developing circuit applications. In microcontroller environment we can use these FPAA circuit in different useful application as an predictive-, and a characteristic prediction circuit solution. We describe a control solution of an industrial machine control as a demonstration of applicability of microcontroller and FPAA circuit cooperation.
\end{abstract}

\section{INTRODUCTION}

The programmable analog circuit $^{1}$, can be used universally understood by the program configured for circuit. (Fig. 1).

FPAAs can be used for the realization of different functional units, circuits, circuit elements. These circuits can be used effectively in applications where the low electric power, the lower development and component cost, the effective electronic CAD possibility are important.

The advantage of FPAAs in the field of faster and more economical circuit planning is significant. It is beneficial in self-developing circuit applications, in neural networks, in signal conditioning, in filters, in fuzzy controls and highfrequency applications. According to other approaches FPAAs serve the linear and non-linear implementation of the analog system and the scalability of the application to be realized. Yet the above mentioned advantages are not obvious since it is very difficult to make a user-friendly FPAA and environment.

It has been suggested that the mixed-signalled architecture should be constructed duplicated, thus this circuit will be suitable for the realization of configurable, self-learning processes, algorithms by reprogramming occurring in the background .

The vast majority of FPAA applications allows the user to use the analog circuit in accordance with the required function taking advantage of the reconfiguration possibility. A further advantage of FPAAs is the simple embedding in bigger hybrid and digital systems.

\footnotetext{
${ }^{1}$ Field Programmable Analog Array, (FPAA)
}

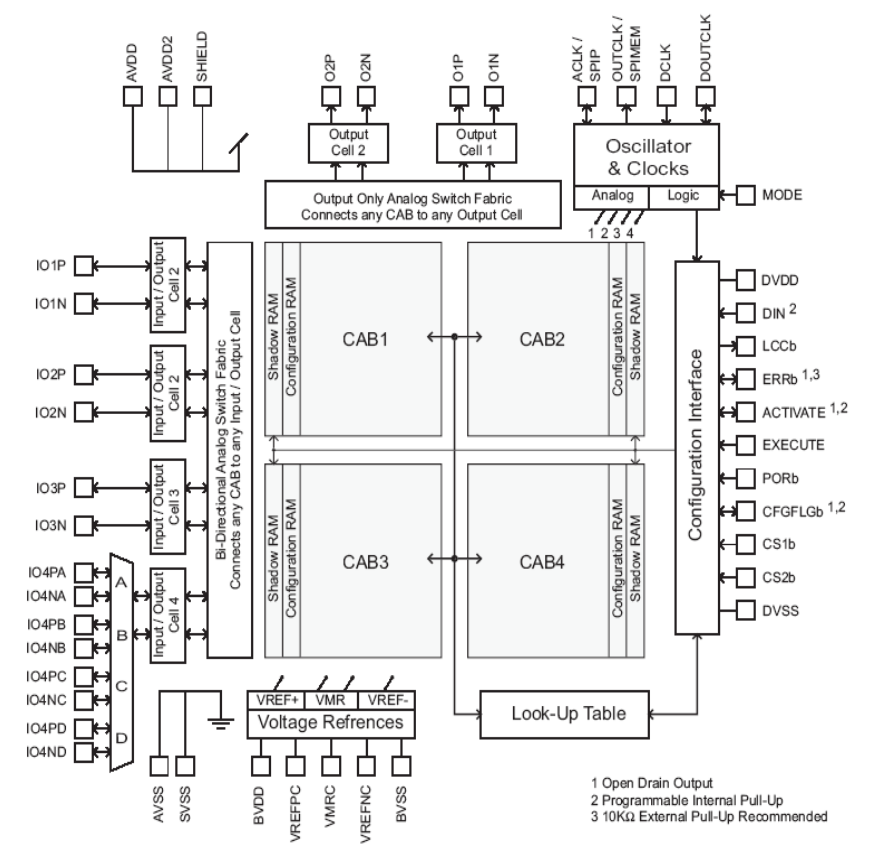

Fig. 1. Internal structure of most popular Anadigm's FPAA with the four configurable analog blocks.

The developments aim at meeting the big analog processing requirements in the field of high integrated FPAA devices in which there are too complex programmable applications: high-order filters (Fourier-processor), adaptive filter systems, vector-multiplier, matrix-multiplier.

In multi-value logics, in neural networks, in mixed-signal processor digital and analog circuits in which the traditional microprocessor is integrated onto a silicon chip with lowperformance analog circuit elements, further applications are offered. The smaller geometrical size, the fewer outputs, the cheaper mounting, the specifically smaller dissipation falling on of one volume unit are among the advantages.

Developments to be realized in the field of programmable analog circuits are as follows: speed, accuracy, digital noise, analog noise, performance, resource allocation possibility (capacity of FPAAs, component-level configurability), source usability, effective architectures, development environment ser- 
vices, macros, simulation, dynamic reprogramming.

Research into the applications of programmable analog circuits, the spread of their application possibilities are to be solved. [1] [2] [3] [4] [5] [6] [7] [8]

\section{Characteristic Prediction in ANAlog Circuit}

Programmable analog circuits, an important area of research on the use of analog and hybrid electronic circuit technology. Particularly interesting is the field where, for a device already present in the microcontroller may be any co-operation between the two cardinal devices. The microcontroller and FPAA such co-operation allows the "embedded hybrid system" tool for using. In this article, beyond the theoretical approach, a practical application being introduced, which realizes the characteristic prediction [9].

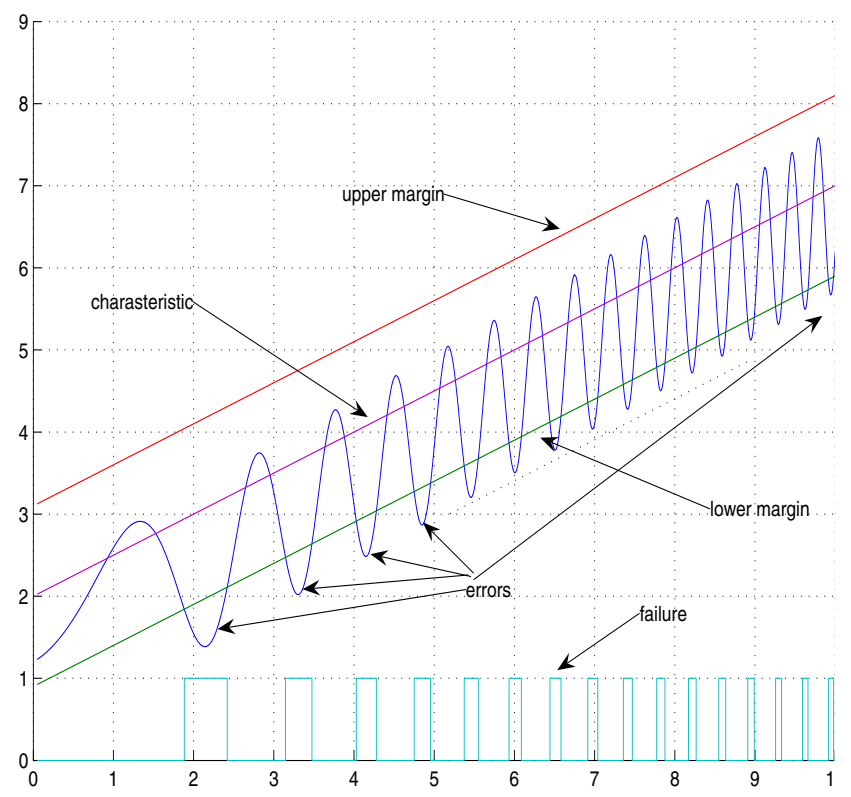

Fig. 2. Making robust electronic circuits with a characteristic prediction.

The diagram of figure 2 explains the characteristic prediction is observed. The predictor determines the analog characteristics of the expected value and the error range. If the analog signal will fall outside the range, the error signal is produced. The error signal, optionally used in the circuit re-configuration or other signals to generate.

On the figure 3 carries out error detection. In this case a predictor circuit $(f)$ maps the "good operation" values from the input signal $\left(U_{i n}\right)$. Thus the output signal of the current circuit $(F)$ is compared with the output of the characteristic predictor circuit. The comparator operates as a windowcomparator and checks the in-range character of the circuit output value according to (4.3), where $\left(U_{c l}\right)$ is the lower comparing threshold voltage, $\left(U_{c o}\right)$ is the upper comparing thresh-old voltage.

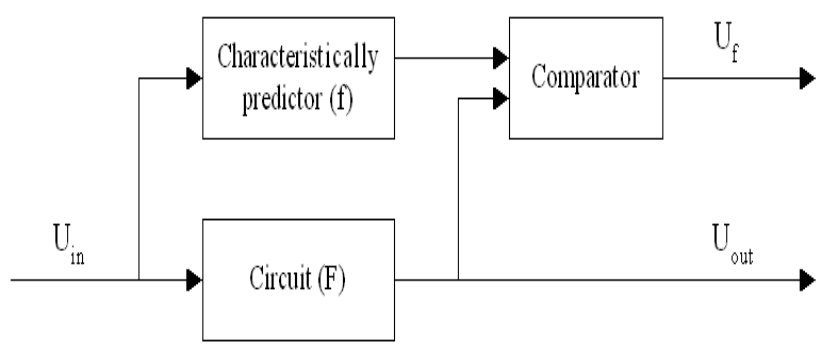

Fig. 3. Realization of a characteristic predictor circuit in a robust system.

$$
U_{f}=\left\{\begin{array}{ccc}
U_{f} & \text { if } & U_{c l}>\left(f\left(U_{i n}\right)-\mathbb{F}\left(U_{i n}\right)\right) \geq U_{c o} \\
0 & \text { if } & U_{c l} \leq\left(f\left(U_{i n}\right)-\mathbb{F}\left(U_{i n}\right)\right)<U_{c o}
\end{array}\right.
$$

where: $U_{c l}$, lower threshold voltage of comparator, $U_{c o}$ upper threshold voltage of comparator, what calculated of the output of $f$ circuit, and the hysteresis $(h)$ according of equations 2,3 .

$$
U_{c l}=f\left(U_{i n}\right)-\frac{h}{2},
$$

and,

$$
U_{c o}=f\left(U_{i n}\right)+\frac{h}{2} .
$$

In case of an error, the further usage of the output signal, the selection, change and correction of the actual circuit and that of the reference circuit are new, challenging system technological tasks [10] [11].

\section{Characteristic Prediction With FPAA}

The error detection is one possible solution to the characteristic predictor application. This, a programmable analog circuit and microcontroller implemented decomposition is shown in the figure 4 .

The programmable analog circuit $\left(\mathbb{F}_{F P A A}\right)$ input receives an input signal $\left.\left(A_{(} a\right)\right)$, output $\left.\left(A_{(} a\right)\right)$ the system output signal to (4) according to the description,

$$
A_{\text {out }}=\mathbb{F}_{F P A A}\left(A_{\text {in }}\right),
$$

where: $\mathbb{F}_{F P A A}$ circuit function of programmable analog circuit.

The $A_{\text {in }}$ input signal the of $(A D C)$ too, which generate $D_{b e}$ signal (5),

$$
D_{b e}=\mathbf{d}\left(A_{i n}\right) .
$$

Here $\mathbf{d}$ is the transfer characteristic of analog-digital converter. 


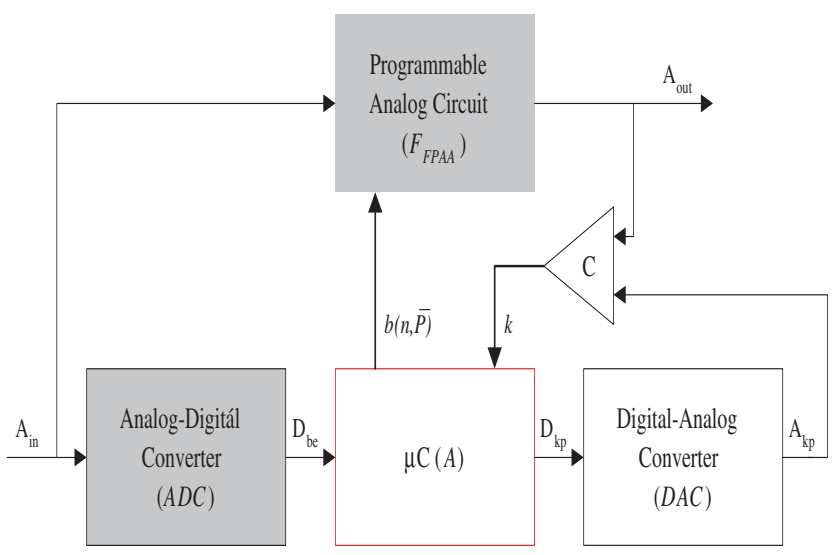

Fig. 4. Solution of analog error detection with a characteristic prediction in microcontroller environment. The blocks are shown in gray in the microcontrollers may be located.

The $D_{b e}$ signal goes to the input of microcontroller $(\mu C)$ which from this value create, by the characteristic prediction, the $D_{k p}$ signal (6),

$$
D_{k p}=\alpha_{k p}\left(D_{b e}\right)
$$

where: $\alpha_{k p}$ characteristic prediction part of the the firmware of microcontroller $(\boldsymbol{A})$, in short; $\alpha_{k p} \in \mathbf{A}$.

The digital-analog converter the $D_{k p}$ signal transforms to analog signal, according of (7),

$$
A_{k p}=\mathbf{a}\left(D_{k p}\right)
$$

where: a the transfer characteristic of digital-analog converter.

The hysteresis comparator cooperates $(C)$ the $A_{k i}$ and $A_{k p}$ signals (8) namely:

$$
k=\left\{\begin{array}{lll}
\mathbf{0} & \text { if } & \left(A_{k p}+\frac{h}{2}\right)>A_{\text {out }} \geq\left(A_{k p}-\frac{h}{2}\right) \\
\mathbf{1} & \text { if } & \left(A_{k p}+\frac{h}{2}\right) \leq A_{\text {out }}<\left(A_{k p}-\frac{h}{2}\right)
\end{array}\right.
$$

Here $h$ the programmable hysteresis value's of comparator.

If $A_{\text {out }}$, defined by the prediction value $\left(A_{k p} \pm h\right)$ is outside the range, the comparator output $((k)$ binary $(1)$, which may for the microcontroller error value. The error causes the microcontroller to the A algorithm re-configure the FPAA circuit $b(\mathbf{n}, \bar{P})$ interface, thereby creating the new circuit function (9):

$$
\mathbb{F}_{F P A A}=\mathbf{A}[b(\mathbf{n}, \bar{P})] .
$$

The (4)-(9) prediction equations, as described in the operating characteristic function formally layout : $\left(A_{k i}, A_{k p}\right) \Rightarrow$ $k \Rightarrow \mathbf{A} \Rightarrow b(\mathbf{n}, \bar{P}) \Rightarrow \mathbb{F}_{F P A A}$, and $\mathbb{F}_{F P A A} \Rightarrow A_{k i}$ over and above $\alpha_{k p} \Rightarrow A_{k p}$, so the feedback is established.

The microcontroller characteristic segment of the program $\left(\left(\alpha_{k p}\right)\right.$, the complexity of the prediction, depending on the accuracy of the required resources (time) can be demanding.
Thus, this procedure is complicated algorithm, only the analog signal $A_{b e}$ ) over frequency limit reduction will be achieved.

The ?? proposed layout of the architecture of reality is much simpler, as indicated in the gray blocks of programmable analog circuit can be formed, the comparator and the digitalanalog converter can be found in most of the microcontroller peripherals [12] [13] [15].

\section{CONCLUSIONS}

The characteristic prediction, and using of re-configuration of programmable analog circuits, to make a robust electronic circuits in which the circuit reliability can be improved greatly. At the same time, or other important aspect, the quality parameter of the analog circuit can be increased.

It is very important that this process lies in the microcontroller's resources are not significant. The microprocessor can only take advantage reconfigure the FPAA when the output of the characteristic predictor gives error signal. In the latter case, even discontinuous relationship can be constructed between the programmable analog circuit and the microcontroller [15].

The proposed solution successfully used the dynamic filter reconfiguration in an EEG unit control [14].

The additional work for the concrete implementation of circuit applications, the optimal algorithm design processes, self-improving solutions to refine the target.

\section{REFERENCES}

[1] A. S. Deese and C. O. Nwankpa. Circuit theoretical analysis of reconfigurable analog load emulation circuit. http://www.ewh.ieee.org/conf/powertech.

[2] R. S. Muller and T. I. Kamins. Device Electronics for Integrated Circuits. John Wiley and Sons, 1986.

[3] C. Reiser. Optimization of performance of dynamically reconfigurable mixed-signal hardware using field programmable analog array (FPAA) technology. PhD thesis, (1998).

[4] Anadigm the dpASP Company. Dinamically programmable analog signal processing. http://www.anadigm.com/.

[5] S. Zebulum, R, A. Stice, and K. Didier. The design process of an evolutionary oriented reconfigurable architecture. www.coe.uncc.edu/ kdatta/papers/The/20Design/20Process/20o...urable/20Architecture.pdf.

[6] Gy. Györök. Self Organizing Analogue Circuit by Monte Carlo Method. LINDI 2007 International Symposium on Logistics and Industrial Informatics September 13-15, 2007 Wildau, Germany, ISBN 1-4244-1441-5, IEEE Catalog Number 07EX1864C, Library of Congress 2007930060, p. 34-37.

[7] Mitra,S. Introduction to Robust Systems. www.stanford.edu/ subh/robust.html.

[8] Gy. Györök. Functional and Parametrical Self Adjustment in Analog Circuit. SISY 2007 5th International Symposium on Intelligent Systems and Informatics August 24-25, 2007 Subotica, Serbia, ISBN 1-4244-1443-1, IEEE Catalog Number 07EX1865C, Library of Congress 2007930059, p. $67-70$.

[9] Gy. Györök. Programmable Analog Circuit in Reconfigurable Systems. 5th Slovakien - Hungarien Joint Symposium on Applied Machine Intelligence, 2007 January 25-26, Poprad, Slovakia, ISBN 978-963-7154-56-0, p. $151-156$.

[10] Gy. Györök, M. Makó. Self configuration Analog Circuits. XVIIth Kand conference 2006 ,In memoriam Klmn Kand” Budapest Tech Kand Klmn Faculty of Electrical Engineering, 12-14 January 2006, ISBN 9637154 426.

[11] Gy. Györök, M. Makó. Configuration of EEG Input-unit by Electric Circuit Evolution. INES 2005, 9th International Conference on Intelligent Engineering Systems, 2005 September 16-19, 2005 Cruising on Mediterranean Sea, ISBN 0-7803-9474-7, IEEE 05EX1202C. 
[12] Gy. Györök, M. Makó, J. Lakner. Combinatorics at Electronic Circuit Realization. ACTA POLYTECHNICA HUNGARICA 6:(1) pp. 151-160. (2009)

[13] Gy. Györök. The FPAA Realization of Analog Robust Electronic Circuit. IEEE Internacional Conference on Computational Cybernetics: ICCC 2009. Palma de Mallorca, Spanyolorszg, 2009.11.26-2009.11.29. Palma de Mallorca: pp. 1-5. Paper 10. (ISBN:978-1-4244-5311-5).

[14] Gy. Györök, M. Makó. Configuration of EEG Input-unit by Electric Circuit Evolution. INES 2005, 9th International Conference on Intelligent Engineering Systems, 2005 September 16-19, 2005 Cruising on Mediterranean Sea, ISBN 0-7803-9474-7, IEEE 05EX1202C.

[15] Gy. Györök. The FPAA Realization of Analog Robust Electronic Circuit. IEEE Internacional Conference on Computational Cybernetics: ICCC 2009. Palma de Mallorca, Spanyolorszg, 2009.11.26-2009.11.29. Palma de Mallorca: pp. 1-5. Paper 10. (ISBN:978-1-4244-5311-5). 